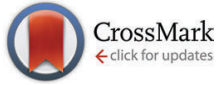

Cite this: J. Mater. Chem. C, 2016 , 4, 5370

Received 10th March 2016, Accepted 25th April 2016

DOI: $10.1039 / c 6 t c 01029 f$

www.rsc.org/MaterialsC

\title{
Poly-(3-hexylthiophene) bottlebrush copolymers with tailored side-chain lengths and high charge carrier mobilities $\uparrow$
}

\author{
C. David Heinrich and Mukundan Thelakkat*
}

A series of well-defined poly(3-hexylthiophene) (P3HT) grafted bottled brushes PS-g-P3HT with different $\mathrm{P} 3 \mathrm{HT}$ chain lengths were synthesized by a grafting-to approach using nitroxide mediated controlled radical polymerization. The influence of the side-chain length on the optical, thermal and electronic properties of these polymers is systematically studied and compared with the corresponding linear P3HTs. It can be shown that the optical, structural and electronic properties of the brushes depend heavily on the side chain lengths. The results of DSC, UV-Vis and AFM measurements reveal two important things. The brush polymers with low molecular weight P3HT side-chains do not crystallize and therefore exhibit very poor electronic properties. With an increase of the side-chain length, highly crystalline materials are obtained and a brush with a high molecular weight $\left(144 \mathrm{~kg} \mathrm{~mol}^{-1}\right)$ carrying long P3HT side chains $\left(17000 \mathrm{~g} \mathrm{~mol}^{-1}\right)$ is necessary to obtain crystalline lamellar structures and the best charge transport properties. For the first time P3HT brush polymers are reported that can match the excellent electronic properties of their linear counterparts in the range of $10^{-2} \mathrm{~cm}^{2} \mathrm{~V}^{-1} \mathrm{~s}^{-1}$. Additionally, the brush polymers exhibit better thin film stability at elevated temperatures compared to linear P3HTs.

\section{Introduction}

Bottlebrush polymers consist of polymeric backbones which are densely grafted with regularly spaced side-chains. A high grafting density forces the backbone into an extended chain through steric repulsion. ${ }^{1,2}$ Moreover, the densely packed side chains cause the polymer backbones to be stiffened and the persistence length of the brush chain increases with increasing side chain density. Therefore this architecture leads to unique material properties. Thus, bottlebrush polymers have far higher polymer chain mobilities than linear polymers with comparable molecular weights due to the absence of chain entanglements. ${ }^{3}$ Therefore, bottlebrush polymers with conjugated, electronically active poly(3-hexylthiophene) (P3HT), which can combine the benefits of the brush architecture and the good electronic properties of linear P3HT, should be interesting materials for organic electronics. Surprisingly, there are no reports on charge carrier mobilities in semiconductor polymer brushes.

In contrast to most other polymeric organic semiconductors, the synthesis of linear P3HT can be conducted in a highly

Applied Functional Polymers, Macromolecular Chemistry I, University of Bayreuth, Universitätsstr. 30, 95440 Bayreuth, Germany.

E-mail: mukundan.thelakkat@uni-bayreuth.de

$\dagger$ Electronic supplementary information (ESI) available. See DOI: 10.1039/ c6tc01029f controlled manner. The Kumada catalyst transfer polymerization which was independently reported by McCullough et al. ${ }^{4,5}$ and Yokozawa et al. ${ }^{6,7}$ allows the synthesis of polymers with defined molecular weights and controlled end groups. ${ }^{8,9}$ In addition, very high molecular weights can be easily achieved in bottlebrushes which may have advantages in terms of mechanical properties, especially in thin film applications. The correlation of molecular weight and mechanical properties is well-studied in linear semiconductor polymers, whereas very little is known about semiconductor bottlebrushes. ${ }^{10}$ Thus the brush architecture can improve the film stability even at high temperatures against delamination simply by the inherent increase in the molecular weight of these systems. Another interesting and important property of chain orientation/alignment might also be positively influenced by this architecture. It was proposed that in organic photovoltaics (OPV), a nanoscale phase-separated donor-acceptor diblock copolymer could have ideal microstructures that could provide optimal pathways for charge collection. ${ }^{11}$ Such a microphase separated system must be preferentially vertically aligned to the substrate; otherwise the structure would consist of effective charge blocking layers. As a first breakthrough the synthesis of microphase separated fully conjugated polymers could be reported ${ }^{12}$ but the alignment of such a system is not yet accomplished. However, thin films of diblock copolymer bottlebrushes were found to form vertical aligned microstructures without the need of difficult post-processing and alignment procedures. ${ }^{13}$ 
Therefore, donor-acceptor brush block copolymers may be possible candidates to solve this issue. This motivated us to investigate in detail the opto-electronic properties concerning P3HT bottlebrushes. At first, it is of course imperative to synthesize P3TH brushes in a well-controlled manner and to study those alone in order to investigate what design rules are necessary to retain or improve the electronic properties of the donor component. There are several examples of surface grafted P3HT brushes synthesized either by grafting-from ${ }^{14,15}$ or grafting-to ${ }^{16,17}$ processes but the synthesis of P3HT bottlebrushes has been only reported a few times up to now. In 2013 Lin et $a .^{18}$ reported the synthesis of PS- $g$-P3HT by grafting P3HT onto a polystyrene backbone by CuAAc click chemistry. P3HT bottlebrushes were also reported by grafting-from ${ }^{19}$ and grafting-through polymerization. ${ }^{20,21}$ For the grafting-through route exo-norbornyl-functionalized $\mathrm{P} 3 \mathrm{HT}$ macromonomers were polymerized by controlled ring opening metathesis polymerization (ROMP). The molecular weights of these P3HT side-chains were relatively low, in the range of only 2000 to $11000 \mathrm{~g} \mathrm{~mol}^{-1}$ (as measured by SEC). Moreover, up to now, no comparative study has been conducted to understand the structure-property relationship, which correlates the length of $\mathrm{P} 3 \mathrm{HT}$ side chains and charge carrier mobility of these systems and compares them with those of linear polymers. Even though OFET devices with linear P3HT as semiconductors have already delivered charge carrier mobilities of up to $0.1 \mathrm{~cm}^{2} \mathrm{~V}^{-1} \mathrm{~s}^{-1}$, no hole mobility data are available for P3HT brushes at all. ${ }^{22-26}$ We hypothesized that in order to obtain bottlebrush polymers containing P3HT, with properties that meet the requirements for applications in OFET or OPV devices, we would need a higher molecular weight of the P3HT side-chains than previously reported.

Several groups reported a correlation between the molecular weight of linear P3HT and the charger carrier mobility of OFET devices. ${ }^{26-28}$ Generally, the measured charge carrier mobilities increased by several orders of magnitude when the molecular weight P3HT was increased. Zhang et al. reported a distinct correlation between the molecular weight of the nanostructure of P3HT and the charge carrier mobility of OFET devices. ${ }^{29}$ A similar correlation was observed by Singh et al. for SCLC devices. ${ }^{30}$ Both reports give indications of an optimum absolute molecular weight of P3HT, in terms of charge carrier mobility, between 10000 and $15000 \mathrm{~g} \mathrm{~mol}^{-1}$. The electrical properties of P3HT are dependent on the polymer's ability to crystallize and the size of the crystalline nanofibrils. AFM and XRD measurements show that the long period of P3HT nanofibers increases as P3HT becomes longer. The long period and simultaneously the charge carrier mobility reach a plateau when the P3HT chains are so long that chain-folding occurs. ${ }^{30}$ If a similar dependence of charge transport on molecular weight exists in the case of bottlebrush polymers as well, then previously reported studies were ignoring this important point of having polymers with a sufficient molecular weight of the side-chains. A previous study by Wong et al., ${ }^{21}$ varied the side-chain length, but also changed the molecular weight of the backbone making an investigation regarding the influence of the length of P3HT side-chains for a given backbone length difficult.
The aim of our study was therefore to elucidate the influence of the chain lengths of $\mathrm{P} 3 \mathrm{HT}$ in brush polymers on charge carrier mobility without varying the molecular weight of the backbone. This is highly required to understand the correlations of optical and electronic properties with the molecular weight of P3HT brushes. We demonstrate that brush polymers can have comparable optical and electronic properties and superior film stability as compared to linear P3HT, if a higher molecular weight P3HT is grafted.

\section{Experimental section}

\section{Materials and methods}

All commercial reagents were, unless otherwise noted, used without further purification. The linear polymers P3HT 1-4, $N$-t-butyl-O-[1-[4-(chloromethyl)-phenyl]ethyl]- $N$-(2-methyl-1-phenylpropyl)hydroxylamine, ${ }^{31}$ 2,2,5-trimethyl-4-phenyl-3-aza-hexane3-nitroxide ${ }^{31}$ were synthesized in our group according to a published procedure. The synthesis of other polymers reported is outlined in the ESI. $\dagger^{1} \mathrm{H}-\mathrm{NMR}(300 \mathrm{MHz})$ spectra were recorded on a Bruker AC 300 spectrometer and calibrated to the solvent peak $\left(\mathrm{CDCl}_{3} \delta=7.26 \mathrm{ppm}\right)$. Fourier transform infrared (FTIR) spectra were recorded on a Perkin Elmer Spectrum 100 FTIR spectrometer in the attenuated total reflection (ATR) mode. Size exclusion chromatography (SEC) measurements were carried out in THF with two Varian MIXED-C columns $(300 \times 7.5 \mathrm{~mm})$ at room temperature and at a flow rate of $0.5 \mathrm{~mL} \mathrm{~min}^{-1}$ using a UV (Waters model 468) detector with $254 \mathrm{~nm}$ wavelength. The SEC values were calibrated with polystyrene as the external standard and 1,2-dichlorobenzene as the internal standard. MALDI-TOF mass spectra were recorded on a Bruker Reflex III with dithranol as the matrix. Differential scanning calorimetry measurements were performed on a Mettler Toledo DSC 2, calibrated with indium and zinc at a heating rate of $10 \mathrm{~K} \mathrm{~min}^{-1}$ under continuous nitrogen flow.

Absorption measurements were carried out on a JASCO V-670. Solutions containing $\mathrm{CHCl}_{3} /$ EtOAc mixtures were used at concentrations of $0.02 \mathrm{mg} \mathrm{mL}^{-1}$. Different solutions were prepared by adding $\mathrm{CHCl}_{3}$ and EtOAc to a polymer stock solution in $\mathrm{CHCl}_{3}$ with a concentration of $0.2 \mathrm{mg} \mathrm{mL}{ }^{-1}$. Polymer films for optical characterization were prepared by spin casting on ODTS treated glass substrates (the substrates were prepared in an identical procedure as the OFET devices) from $6 \mathrm{mg} \mathrm{mL}^{-1}$ chloroform solutions. Atomic force microscopy images were recorded in the intermittent contact mode on a Dimension 3100 Nanoscope V with a Nanoscope V controller and a hybrid closed loop XYZ tip scanner $(5120 \times 5120$ pixels $)$.

\section{Device preparation and characterization}

Bottom gate/bottom contact organic field effect transistors (OFET Gen4) were purchased from Fraunhofer IPMS. N-doped silicon (doping at the surface $n \approx 3 \times 10^{17} \mathrm{~cm}^{-3}$ ) was used as the surface and gate electrode. The dielectric consists of a $230 \pm 10 \mathrm{~nm}$ layer of silicon oxide. Each substrate consisted of 16 devices with a constant channel width of $10 \mathrm{~mm}$ and a 
varying channel length of $2.5-20 \mu \mathrm{m}$. The source and drain electrodes were a $30 \mathrm{~nm}$ thick gold layer on a $10 \mathrm{~nm}$ ITO adhesion layer. The devices were prepared by cleaning in acetone and subsequently in iso-propanol in an ultrasonic bath for $10 \mathrm{~min}$, followed by $15 \mathrm{~min}$ treatment in an ozone oven at $50{ }^{\circ} \mathrm{C}$ and subsequent silanization by $45 \mathrm{~min}$ treatment in a bath of $1 \mathrm{wt} \%$ octadecyltrichlorsilane (ODTS) in toluene at $60{ }^{\circ} \mathrm{C}$. The devices were rinsed with toluene and iso-propanol and dried. Thin polymer films were spin cast from $6 \mathrm{mg} \mathrm{mL}^{-1}$ chloroform solutions at a spinning speed of $4000 \mathrm{rpm}$ under ambient conditions. All devices were stored and measured under a nitrogen atmosphere. The $I V$-characteristics were measured using an Agilent B1500 semiconductor parameter analyzer. The devices were annealed at $10 \mathrm{~K}$ above the melting temperature and slowly cooled down $\left(\sim 1 \mathrm{~K} \mathrm{~min}^{-1}\right)$ to room temperature. All annealing experiments were conducted under a nitrogen atmosphere. Using eqn (1) the charge carrier mobilities were calculated from the slope of the $\left(I_{\mathrm{d}}\right)^{0.5}-V_{\mathrm{g}}$ plots.

$$
I_{\mathrm{d}} \approx \frac{W}{2 L} C_{\mathrm{i}} \mu\left(V_{\mathrm{g}}-V_{\mathrm{T}}\right)^{2}
$$

\section{Results and discussion}

\section{Synthesis}

Our study focuses on the influence of the length of the P3HT side-chains on optical and electronic properties while keeping the polystyrene backbone identical. This was done in order to see if a similar trend of the optical, thermal and electronic properties as for linear P3HTs could also be observed for brush polymers. Four conjugated bottlebrush polymers with different P3HT side-chain lengths and having an identical polystyrene backbone were synthesized in a procedure similar to the one published by Lin et al. ${ }^{18}$ We identified the grafting-to process as the ideal synthesis method to obtain highly comparable polymers as one backbone polymer can be used for the whole series of brush polymers.

As shown in Scheme 1, the polymeric backbone of the brush is synthesized first. Poly-(4-chloromethylstyrene) (PS-Cl) is synthesized by nitroxide mediated radical polymerization (NMRP) utilizing a chlorine functionalized derivative of the well-known Hawker initiator. ${ }^{31}$ PS-Cl can be easily converted to poly-(4azidomethylstyrene) PS-N $\mathbf{N}_{3}$ upon substitution with sodium azide. The quantitative conversion can be observed in ${ }^{1} \mathrm{H}-\mathrm{NMR}$ by the shift of the methyl protons adjacent to the chlorine in PS-Cl versus the azide group in PS-N $\mathbf{N}_{\mathbf{3}}$ (see Fig. S1, ESI $\dagger$ ). First $\mathbf{P S}-\mathbf{N}_{\mathbf{3}}$ with a molecular weight of $M_{\mathrm{n}, \mathrm{SEC}}=3600 \mathrm{~g} \mathrm{~mol}^{-1}$ and low $D=1.20$ (Table 1) is obtained and is utilized as the precursor polymer for all click reactions to synthesize the bottlebrush polymers PS- $g$-P3HT. Additionally, four alkyne end functionalized P3HT polymers P3HT-Alkyne 1-4 were synthesized according to an optimized procedure. ${ }^{32}$ Functionalization with the alkyne end groups was analytically proven by MALDI-ToF mass spectrometry (see Fig. S2, ESI $\dagger$ ) MALDI-ToF mass spectrometry is also the most reliable measurement to obtain the absolute molecular weights of P3HT. Molecular weights $M_{\mathrm{n}, \mathrm{MALDI}}$ between $1600 \mathrm{~g} \mathrm{~mol}{ }^{-1}$ for

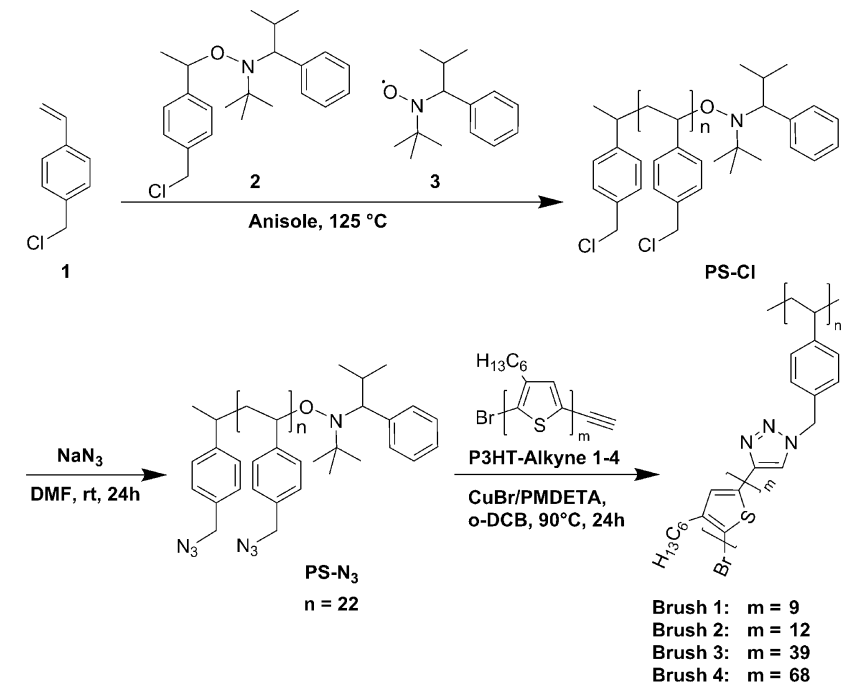

Scheme 1 Scheme of synthesis of poly-4-chloromethylstyrene (PS-Cl) via NMP followed by the polymer analogous substitution of chlorine with sodium azide in order to obtain poly-4-azidomethylstyrene $\left(\mathbf{P S}-\mathbf{N}_{\mathbf{3}}\right.$ ). The conjugated P3HT bottlebrush polymers, Brushes 1-4 were obtained by linking up PS-N $\mathbf{3}$ and four alkyne terminated P3HT polymers (P3HT-Alkyne 1-4) with different chain lengths via CuAAc reaction.

P3HT-Alkyne 1 and $11400 \mathrm{~g} \mathrm{~mol}^{-1}$ for P3HT-Alkyne 4 were obtained. SEC on the other hand is a relative method and it was already reported that the SEC values, when calibrated against polystyrene, are always overestimated for $\mathrm{P} 3 \mathrm{HT} .{ }^{33}$ Table 1 gives a comparison of the $M_{\mathrm{n}}$ values obtained by SEC and MALDI-ToF for the alkyne functionalized polymers P3HT-Alkyne 1-4 and a series of unfunctionalized P3HT polymers P3HT 1-4 used in this study. The linear polymers P3HT 1-4 without an active alkyne end group were later used for comparison with the brush polymers. They were synthesized according to a reported procedure in ref. 8 and are highly comparable to their functionalized counterparts P3HT-Alkyne 1-4 as can be seen from their properties given in Table 1 . The bottlebrushes PS- $g$-P3HT were obtained by a grafting-to process. For this, the alkyne end groups of P3HT-Alkyne 1-4 reacted in a copper catalyzed reaction with the azide groups of PS- $\mathbf{N}_{3}$. The copper catalyzed azide-alkyne reaction (CuAAc) is highly efficient, which is usually conducted at low temperature and is well known as the workhorse of "clickchemistry". ${ }^{34}$ It was nevertheless necessary to conduct the reaction at elevated temperatures of $90{ }^{\circ} \mathrm{C}$ to obtain high conversions. The success of grafting can easily be monitored by SEC. The SEC traces of Brush 3, as one typical example, and the corresponding precursor polymers are shown in Fig. 1a. After the synthesis of the bottlebrush polymer the SEC trace is markedly shifted to high molecular weights. A rather narrow distribution is also an indicator of excellent conversion i.e. high grafting density. A tailing in the low molecular weight region would be otherwise expected if the reaction were incomplete. But this is not observed. A second peak from the P3HT precursor can be observed. This is the case for all synthesized brush polymers because P3HT-Alkyne is always added to the reaction in an excess of 1.1-1.5 equivalents (see Fig. S3a, ESI $\dagger$ ). The raw products could be purified by preparative 
Table 1 Summary of the synthesized polystyrene derivatives PS-Cl and PS- $\mathbf{N}_{3}$, the four different alkyne-functionalized P3HT polymers P3HT-Alkyne 1-4, the resulting bottlebrush polymers, Brushes 1-4, and the unfunctionalized linear polymers P3HT 1-4

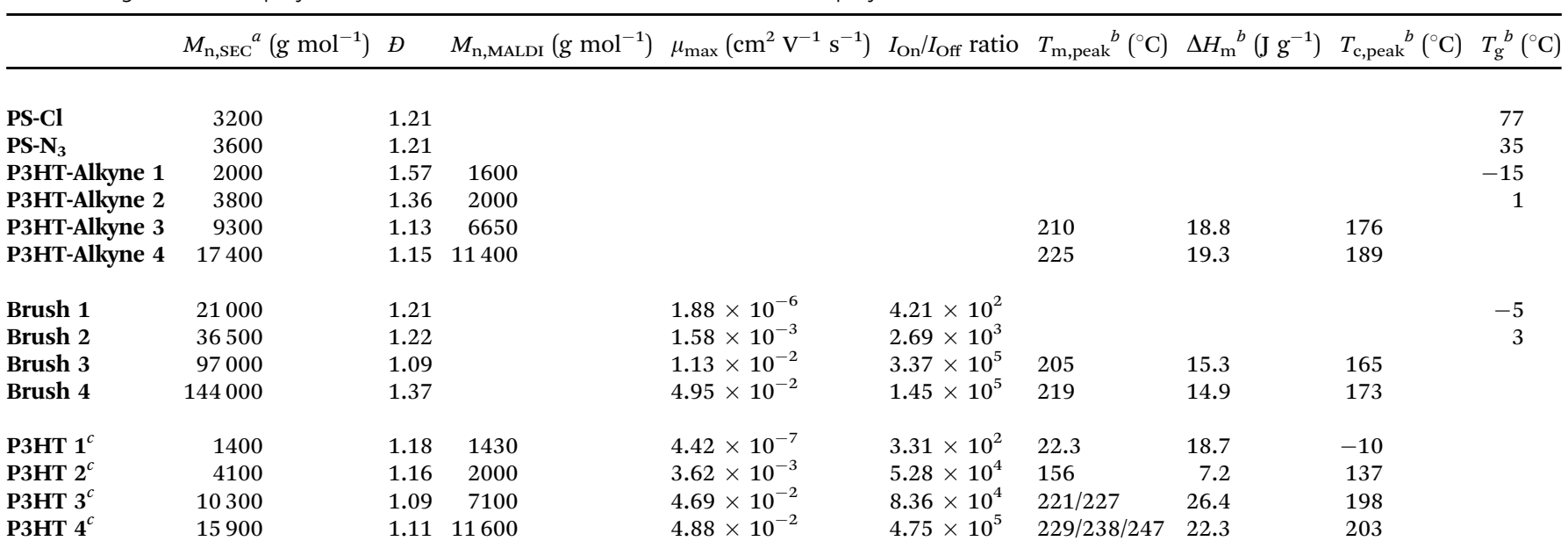

${ }^{a}$ Measured in THF with a polystyrene calibration and $o$-dichlorobenzene as internal standard. ${ }^{b}$ Measured via DSC with a heating/cooling rate of $10 \mathrm{~K} \mathrm{~min}^{-1} .{ }^{c}$ Non-functionalized linear P3HT reference polymers.

a)

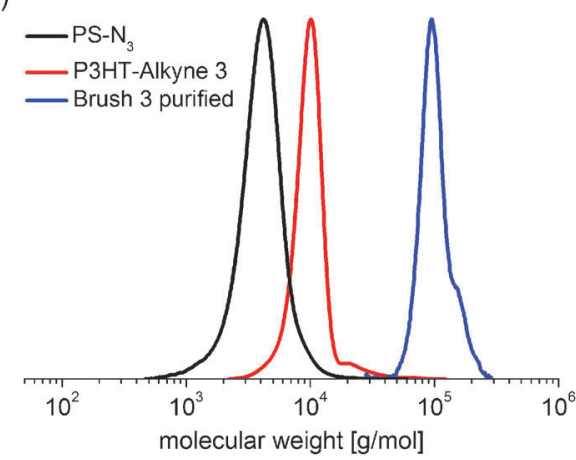

b)

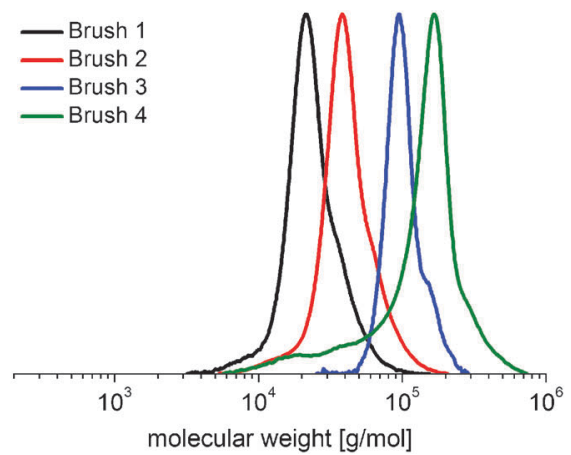

Fig. 1 (a) Evolution of the SEC traces for Brush 3 which is synthesized by the CuAAc of PS- $\mathbf{N}_{\mathbf{3}}$ and $\mathbf{P} 3 \mathrm{HT} \mathbf{3}$ as a typical example; the raw product is purified from the excess of the linear P3HT 3 via preparative SEC; (b) SEC traces of the four purified bottlebrush copolymers Brushes 1-4.

SEC in the case of Brushes 1-3. The solubility of Brush 4 in THF was found to be too low for preparative SEC but most of the residual P3HT-Alkyne could be removed by washing the polymer with THF, which predominantly dissolves the linear polymer. The SEC series of the series of purified bottlebrush polymers are shown in Fig. 1b.

Brush 4 with an $M_{\mathrm{n}, \mathrm{SEC}}$ of $144000 \mathrm{~g} \mathrm{~mol}^{-1}$ having side-chains of $11400 \mathrm{~g} \mathrm{~mol}^{-1}$ in absolute terms (MALDI) is thus the conjugated P3HT bottlebrush polymer with the longest side-chain reported until now. In comparison Wong et al. reported bottlebrushes of P3HT synthesized by ROMP from a P3HT macromonomer with molecular weights of up to only $M_{\mathrm{n}, \mathrm{SEC}}=11200 \mathrm{~g} \mathrm{~mol}^{-1}$, which may correspond to approximately $8000 \mathrm{~g} \mathrm{~mol}^{-1}$ in absolute terms. ${ }^{21}$

The SEC measurements alone are not sufficient to make a statement about the grafting density in the final polymers. In the case of Brushes 1 and 2, ${ }^{1} \mathrm{H}$-NMR spectroscopy can provide this missing information and make it possible to estimate the conversion. By examining the ${ }^{1} \mathrm{H}-\mathrm{NMR}$ of Brush 1 (see Fig. S3b, $\mathrm{ESI} \dagger$ ), we find that the peak of the methyl protons adjacent to the azide in $\mathbf{P S}-\mathbf{N}_{3}$ is shifted from $4.4 \mathrm{ppm}$ to $5.4 \mathrm{ppm}$ due to the formation of triazole after the click reaction. Additionally, a new peak emerges at around $7.6 \mathrm{ppm}$. This peak can be assigned to the only proton of the triazole ring itself. The grafting density can be estimated by comparing the integrals at $4.4 \mathrm{ppm}$ and $5.4 \mathrm{ppm}$. We estimate the grafting density of the polymers, Brushes 1 and 2, where this calculation is possible, to be $85 \%$ for Brush 1 and $90 \%$ for Brush 2. Lin et al. ${ }^{18}$ claimed nearly quantitative grafting of different poly(4-azidomethylstyrene) backbones with a relatively short P3HT-Alkyne $\left(M_{\mathrm{n}, \mathrm{SEC}}=4100 \mathrm{~g} \mathrm{~mol}^{-1}\right)$ without being able to observe the peak at $5.4 \mathrm{ppm}$ or at $7.6 \mathrm{ppm}$ in ${ }^{1} \mathrm{H}$-NMR. The assignment of the peak at $4.4 \mathrm{ppm}$ to the proton at the triazole ring is questionable and is not in agreement with findings in low molecular weight model compounds with a similar chemical structure at the triazole ring. ${ }^{35}$ The polystyrene backbone in the bigger brush polymers, Brushes 3 and 4, on the other hand is already so diluted in relation to P3HT that a quantitative statement about the grafting density is not possible.

\section{Thermal properties}

The thermal properties were examined via DSC measurements. The linear reference polymers are all semicrystalline and the melting and crystallization temperatures increase considerably 
for higher molecular weights. The dependency of the melting temperature on the length of the backbone has been described theoretically for polyolefins ${ }^{36,37}$ and it was shown that P3HT follows the same trend. ${ }^{38,39}$ The small brush polymers on the other hand exhibit a different thermal behavior. The two brushes with very short P3HT chains (Brushes 1 and 2) do not crystallize at all. Both polymers are apparently amorphous and only glass transitions below room temperature are observed $\left(T_{\mathrm{g}}\right.$ of $-5{ }^{\circ} \mathrm{C}$ for Brush 1 and $3{ }^{\circ} \mathrm{C}$ for Brush 2 respectively; see also Fig. S4(a) and (b), ESI $\dagger$ ). We suggest that this is due to the influence of the polystyrene backbone, which in the case of the short brushes makes up for about $8 \mathrm{wt} \%$ of the overall brush polymer, and the very low $T_{\mathrm{m}}$ expected for low molecular weight P3HT. ${ }^{40}$ The connection to the polystyrene backbone greatly reduces the mobility of the P3HT chains. An additional higher glass transition that could be assigned to the polystyrene backbone cannot be observed and it is unclear if the backbone in such a high dilution can vitrify separately. The difference in the glass transition temperatures of the precursor polymers PS-Cl $\left(77^{\circ} \mathrm{C}\right)$ and PS-N $\mathbf{N}_{3}\left(35^{\circ} \mathrm{C}\right)$ also shows that even a relatively small change of the substituents of the polystyrene has a pronounced effect on the thermal properties. A comparison of the P3HT precursors P3HT-Alkyne 1 and 2 with linear P3HT 1 and 2 can help understand the system more. Even the influence of the end groups in very short polymers can be very big. P3HT-Alkyne 1 and 2 for instance are amorphous with a $T_{\mathrm{g}}$ of $-15{ }^{\circ} \mathrm{C}$ and $-1{ }^{\circ} \mathrm{C}$ whereas P3HT 1 and 2 are crystalline. A different behavior can be observed for longer brush polymers, Brushes 3 and 4. In both cases, melting and crystallization of the P3HT side-chains can be clearly observed. In Fig. 2 Brush 4 and the linear reference polymer P3HT 4 are compared as an example. It can be clearly seen that the melting temperature $T_{\mathrm{m}}$ and the enthalpy $\Delta H_{\mathrm{m}}$ as well as the kinetics of crystallization are different for the brushes. The $T_{\mathrm{c}}\left(173{ }^{\circ} \mathrm{C}\right)$ of Brush 4 is about $30 \mathrm{~K}$ lower than that for the linear counterpart P3HT $4\left(203{ }^{\circ} \mathrm{C}\right)$ which is an indication for a kinetically hindered crystallization due to the reduced mobility of the polymer side-chains. Additionally, the enthalpy of phase transition $\Delta H_{\mathrm{m}}$ is reduced from $22.3 \mathrm{~J} \mathrm{~g}^{-1}$ in P3HT 4 to $14.9 \mathrm{~J} \mathrm{~g}^{-1}$ in Brush 4. This corresponds to a reduction of enthalpy by $40 \%$ and therefore cannot be explained by the

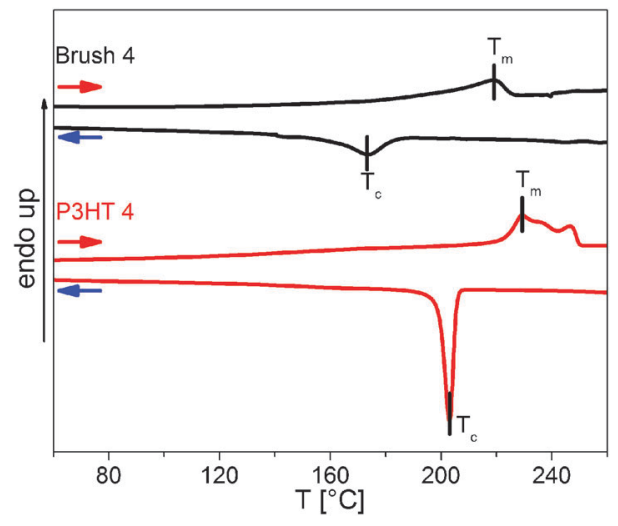

Fig. 2 DSC measurements of Brush $\mathbf{4}$ and the linear equivalent P3HT $\mathbf{4}$ as examples at a heating/cooling rate of $10 \mathrm{~K} \mathrm{~min}^{-1}$. diluting effect of the backbone alone which only makes up 1.5\% of the overall mass of the bottlebrush. This means, that the steric hindrance which is induced through the change in the polymer architecture must be the cause for a reduced overall crystallinity. If the published $\Delta H_{\mathrm{m}}^{\infty}=33 \mathrm{~J} \mathrm{~g}^{-1}$ value for linear P3HT is taken into account, then Brush 4 can be considered to be $45 \%$ crystalline. ${ }^{41}$ The behavior of Brush 3 is comparable to Brush 4. The only difference is a lower $T_{\mathrm{c}}$ and $T_{\mathrm{m}}$ for both linear and brush polymers due to shorter side-chains. Brush 3 also has a very similar degree of crystallinity of $46 \%$.

\section{Optical properties of solutions and films}

Further information about the aggregation properties of the bottlebrush polymers can be extracted from UV-spectroscopy. UV-Vis spectra of either thin films or solutions of linear P3HT can easily be qualitatively analyzed for a possible aggregation of the polymer. According to Spano et al. ${ }^{42}$ P3HT forms weak $\mathrm{H}$-aggregates in thin films or in bad solvents. The aggregates have a distinctly different absorption spectrum in comparison to non-aggregated $\mathrm{P} 3 \mathrm{HT}$ coils giving rise to the possibility of deconvoluting the spectra and even estimating the fraction of aggregates. ${ }^{43}$ The solution spectra of P3TH exhibit one broad peak which shows a bathochromic shift when the polymer mass increases, indicating that the conjugated system grows. This saturates at a certain molecular weight i.e. a further increase of the molecular weight of the polymer will not induce additional changes to the spectra. The saturation value can be observed at around $450 \mathrm{~nm}$ (Fig. S5a, ESI $\dagger$ ). If aggregates are present a bathochromic shift of nearly $100 \mathrm{~nm}$ occurs and three distinct peaks can be observed.

The solution behavior of the bottlebrush polymers was characterized first. The first question was if Brushes 1 to 4 would form non-aggregated solutions or if the large molecular weight and the different architectures of brushes would lead to irreversible aggregates as it was observed in brushes with a polynorbornene backbone. ${ }^{20} \mathrm{UV}$-Vis spectra of the chloroform solutions of all four brush polymers were measured (see Fig. S5a, $\mathrm{ESI} \dagger$ ). All four spectra resemble the spectra of coiled P3HT in solution and aggregation was not observed. The peak maximum shifts as expected with the increase of the P3HT side-chain length from Brushes 1 to 4 from $432 \mathrm{~nm}$ to $454 \mathrm{~nm}$.

We also investigated the influence of the solvent nature in a similar manner as reported for linear $\mathrm{P}_{3} \mathrm{HT}^{44}$ by using mixtures of a good solvent (chloroform) and a bad solvent (ethyl acetate) without changing the overall polymer concentration. As an example the aggregation of Brush 4 in solution is shown in Fig. 3a for increasing amounts of non-solvent ethyl acetate.

The P3HT side-chains in pure chloroform solution are in a non-aggregated state. Adding 15 vol\% EtOAc already induces a big change. Here the spectrum is a superposition of a fraction of coiled P3HT and of the aggregated species. The spectra of the aggregates are characterized by the three peaks at $524 \mathrm{~nm}$, $560 \mathrm{~nm}$ and $608 \mathrm{~nm}$ which can be identified as the 0-2, 0-1 and the $0-0$ transition. According to Spano et al. the ratio of the absorbance of the $0-0$ peak and the $0-1$ peak can be used to determine the quality or the degree of aggregation. A high $0-0$ 

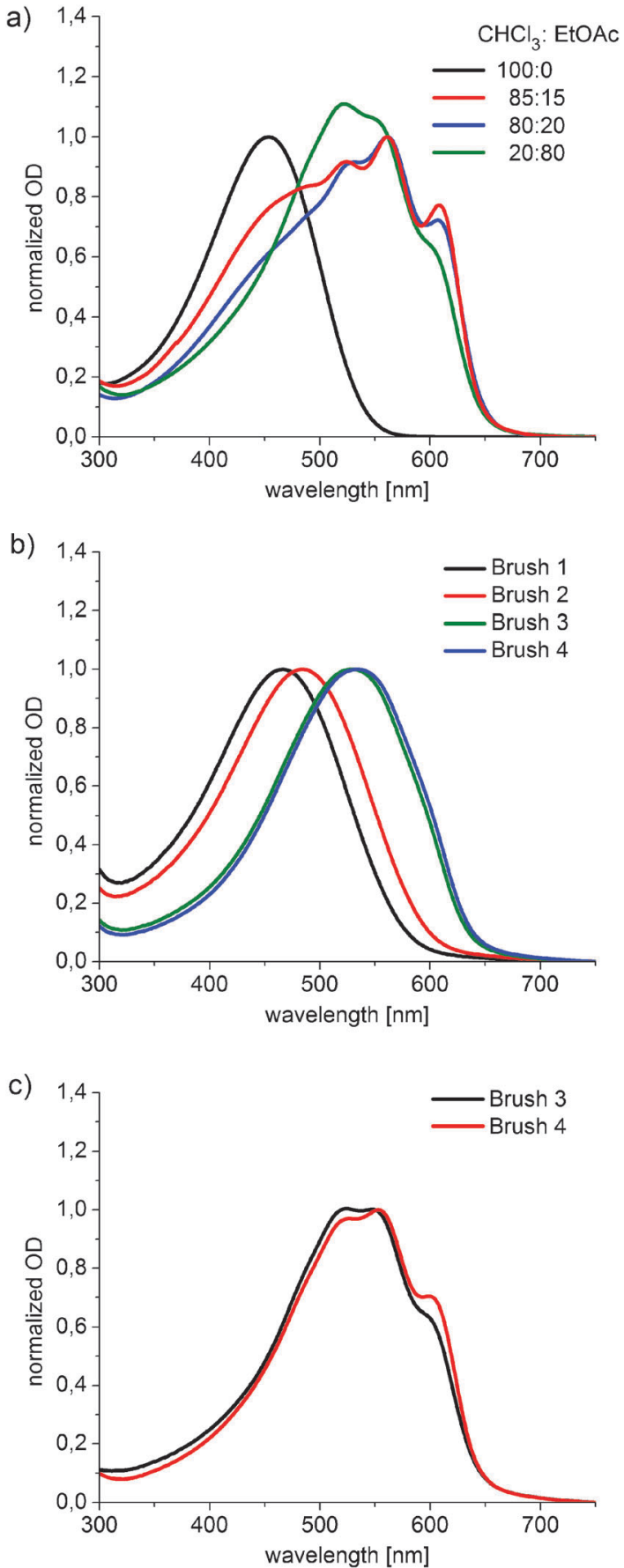

Fig. 3 (a) UV-Vis spectra of Brush 4 in a mixture of a good solvent $\left(\mathrm{CHCl}_{3}\right)$ and a bad solvent (EtOAc) exhibiting pronounced aggregation with increasing EtOAc content; (b) UV-Vis spectra of the brush polymers, Brushes 1-4, in as-cast films and (c) of Brushes $\mathbf{3}$ and $\mathbf{4}$ after melt crystallization.

to $0-1$ ratio is an indicator of a higher order. ${ }^{43}$ A qualitative examination of the spectra of Brush 4 for increasing amounts of non-solvent shows that while the fraction of aggregates increases (as evidenced by the decrease of the peak from the coiled P3HT at $454 \mathrm{~nm}$ ), the aggregates must be less ordered as the relative intensity of the $0-0$ absorption decreases with respect to the $0-1$ transition. The ratio of $0-0$ to the $0-1$ absorption decreases from $A_{0-0}: A_{0-1}=0.78$ for $15 \mathrm{vol} \%$ of the nonsolvent ethyl acetate to $A_{0-0}: A_{0-1}=0.59$ when the ethyl acetate amount is increased to $80 \mathrm{vol} \%$ (see Fig. 3a). Brush 3 also forms aggregates in solution and an increase of the fraction of aggregates can be observed with increasing content of the non-solvent ethyl acetate. We cannot observe a distinct change in the shape of the spectra in this case (see Fig. S5b, ESI $\dagger$ ). The ratio of $0-0$ to the $0-1$ absorption increases only slightly from $A_{0-0}: A_{0-1}=0.55$ for $20 \mathrm{vol} \%$ of the nonsolvent ethyl acetate to $A_{0-0}: A_{0-1}=0.55$ with an ethyl acetate content of $80 \mathrm{vol} \%$. That means, that in contrast to Brush 4 the quality of the aggregates in Brush 3 does not change with the solvent quality. A similar investigation of the short brushes, Brushes $\mathbf{1}$ and 2, was conducted but no spectral changes for the different solvent mixtures were detected. Brushes $\mathbf{1}$ and 2 can therefore not aggregate at all even in $90 \mathrm{vol} \%$ ethyl acetate solution. This result is also in agreement with bulk DSC measurements, which showed that these polymers could not crystallize.

We were further interested in the thin film properties of brush polymers. To be able to compare the results with the OFET measurements later, we conducted the following experiments on comparable films. We ensured that the substrate had the same properties as in the OFET devices by the hydrophobization of the surface with octadecyltrimethoxysilane (ODTS) and prepared the films by spin casting chloroform solutions. In Fig. $3 \mathrm{~b}$, the UV-Vis spectra of all four brushes are shown directly after spin casting. The spectra of the films of Brushes 1-4 are unstructured but a bathochromic shift can be observed in comparison to the corresponding spectra of the chloroform solutions of these polymers. The peak maxima of the small brushes, Brushes 1 and 2, are red-shifted by $32 \mathrm{~nm}$ and $45 \mathrm{~nm}$ in thin films. The shift of the maxima of Brushes 3 and 4 in thin films is larger with approximately $80 \mathrm{~nm}$ for both polymers. The onset of absorption at around $650 \mathrm{~nm}$ for Brushes $\mathbf{3}$ and $\mathbf{4}$ is the same as expected for aggregated linear P3HTs. The films of these two brush polymers (Brushes $\mathbf{3}$ and 4) are therefore to some extent aggregated. If compared to linear P3HT 1-4 a different behavior can be observed (Fig. S5c, ESI $\dagger$ ). While the spectrum of the as-cast film of P3HT 1 resembles the non-aggregated solution spectrum in chloroform, the as-cast films of P3HT 2-4 with higher molecular weights exhibit structured spectra in which three vibrational bands can be observed.

Spin casting polymer films from low boiling solvents such as chloroform leads to films with low crystallinity as the film solidifies very fast. All polymer films were therefore also meltcrystallized in order to have maximum aggregation due to improved crystallization. However, the films of the linear polymers P3HT 1-4 and the small brush polymers, Brushes 1 and 2, were not stable on the highly hydrophobic surface and showed dewetting behavior. Brushes 3 and 4 on the other hand, formed high quality films due to their high molecular weight that showed no signs of dewetting. The spectra of the melt crystallized films of Brushes 3 and $\mathbf{4}$ (Fig. 3c) do now show structured curves expected for well-aggregated P3HT. While the as-cast films of Brushes 3 and 4 were nearly identical to their order of aggregation, the ratio of the $0-0$ band at around $605 \mathrm{~nm}$ to the $0-1$ band at $560 \mathrm{~nm}$ for the melt crystallized film of Brush 4 is higher $\left(A_{0-0}: A_{0-1}=0.70\right)$ as compared to Brush $3\left(A_{0-0}: A_{0-1}=0.62\right)$. 
This is, as mentioned before, an indicator of a higher order of the melt-cast film of high molecular weight Brush 4.

\section{Atomic force microscopy}

A linear P3HT is known to form lamellar crystallites which can be observed by either atomic force microscopy (AFM) ${ }^{30,45}$ or transmission electron microscopy (TEM). ${ }^{46}$ For grafted P3HT bottlebrush polymers, no optical characterization has been reported in the literature. We therefore investigated Brushes 3 and 4, where we already observed crystallization in DSC and UV-Vis, by AFM. Fig. 4 shows the observed structures for the linear P3HT (P3HT 4) in comparison to the brush polymers with similar side-chains (Brush 4). A highly ordered lamellar structure is observed for the linear polymer. Interestingly, the brush polymer forms very similar structural features, but with less order. The long period, that means the thickness of the crystalline lamellae (bright color) and the amorphous part (dark color) combined, can be roughly estimated to be $25 \mathrm{~nm}$ for both polymers. This is in the range of the contour length of stretched P3HT chains with a molecular weight of around $12000 \mathrm{~g} \mathrm{~mol}^{-1}$ (MALDI). This is in good agreement with the reported values of Singh et al., where extended P3HT chains were observed in melt-crystallized samples up to molecular weights of $12000 \mathrm{~g} \mathrm{~mol}^{-1}$ (MALDI). ${ }^{30}$ This is the first report on the brush polymer that crystallizes in a similar manner to its linear counterpart in the high molecular weight range. Though the AFM picture of the brush shows a lower order as well as a smaller length of the crystalline lamellae. This is consistent with the already observed differences between the linear and the brush polymers that we discussed in DSC and UV-Vis measurements and is yet another indication of a hindered crystallization due to mobility restrictions induced by the immobilization of one chain end on the polystyrene backbone. In the investigation of Brush 3 and its linear counterpart P3HT 3, on the other hand, we could not observe a clear structure for the brush (see Fig. S6, $\mathrm{ESI} \dagger$ ). This is unexpected due to the otherwise similar characteristics of aggregation (UV-Vis, DSC) in comparison to Brush 4.

\section{Charge carrier mobilities}

The influence of the side-chain length on the charge carrier mobilities of P3HT brush polymers was investigated in organic field effect transistors (OFETs). We prepared devices in a bottom gate/bottom contact configuration. The detailed description of the preparation and device structure is given in the Experimental section. Herein we compare the charge carrier mobilities of Brushes 1-4 with those of the linear counterparts P3HT 1-4. We show the p-type output curves and the p-type transfer curves of melt crystallized Brush 4 (Fig. 5a and b) as a sample with the highest measured mobility within the series of tested polymers. The OFET device with the melt crystallized Brush 4 as an active material reached a hole carrier mobility of $\mu_{\mathrm{h}}=4.95 \times 10^{-2} \mathrm{~cm}^{2} \mathrm{~V}^{-1} \mathrm{~s}^{-1}$. This value is comparable to the highest values that we could achieve for the linear polymers and it is also in the range of the best reported mobilities for P3HT. ${ }^{25}$ The measurements of the hole mobilities of P3HT 1-4 show a high dependency of the hole mobility on the molecular weight. ${ }^{30}$ An increase of the molecular weight from $1400 \mathrm{~g} \mathrm{~mol}^{-1}$ to $11600 \mathrm{~g} \mathrm{~mol}^{-1}$ (MALDI) increases the hole mobility by five orders of magnitude. Earlier reports suggest that a further increase of the molecular weight above approximately $12000 \mathrm{~g} \mathrm{~mol}^{-1}$ (MALDI) will not increase the charge carrier mobility, measured by SCLC, in the film. ${ }^{30}$ Attempts to further improve the OFET device with linear P3HT by thermal annealing were not successful. The thin films of the linear P3HT polymers were not stable on the hydrophobic surface and showed dewetting behavior. The PS- $g$-P3HT bottlebrushes under investigation also showed a similar dependency of the hole mobilities on the molecular weights of the conjugated P3HT side-chains (see Fig. $5 \mathrm{c}$ ). The measured mobilities of as-cast films of Brushes 1-4 were lower than the as-cast films of the corresponding linear polymers. Thus the decrease in order/crystallinity in brush polymers discussed above negatively influences the charge carrier mobility in the brush polymers to a certain extent. The devices with brush polymers were also thermally annealed above the melting temperature. The polymers, Brushes 3 and 4, were stable even after melt crystallization and a marked increase in the charge carrier mobilities could be measured for both polymers after annealing. Brush 4 thus exhibited the best mobility compared to excellent film stability. These results are in accordance with the UV-Vis measurements which show an increase of the crystallinity after melt crystallization. These results clearly show that PS- $g$-P3HT bottlebrushes with high molecular weight
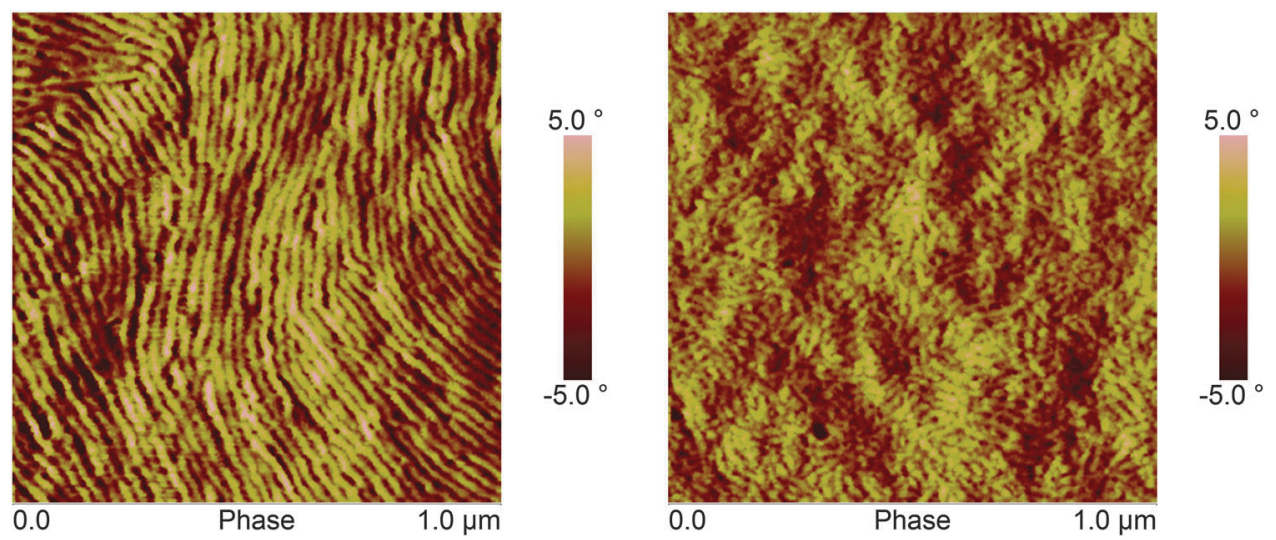

Fig. 4 AMF phase image of P3HT4 (left) and Brush 4 (right) after melt crystallization. 
a)

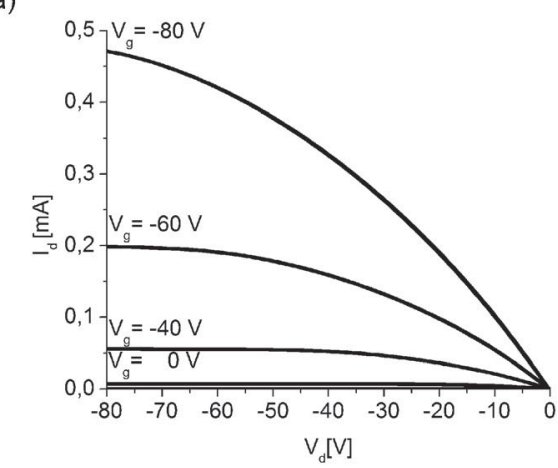

c)

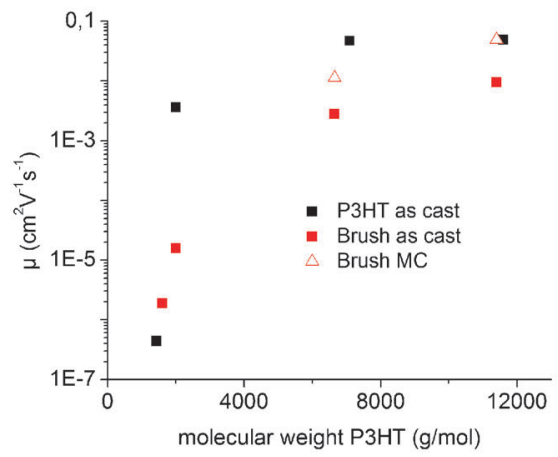

b)

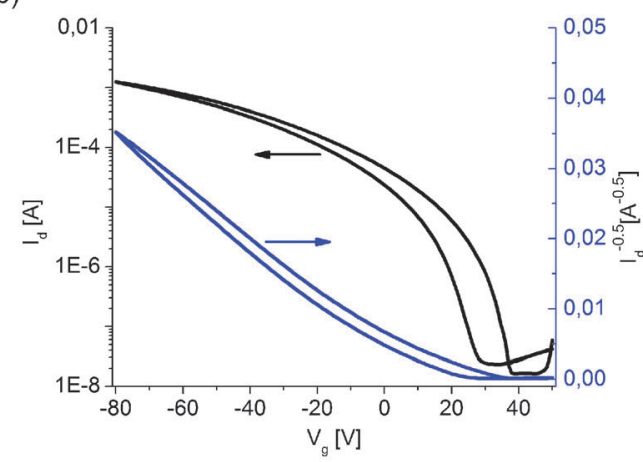

d)

d)

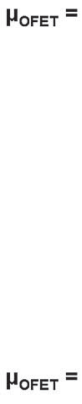

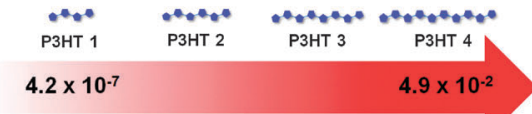

?
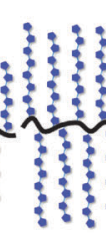

Brush 1

Brush 2

Brush 3

$1.9 \times 10^{-6}$

Fig. 5 (a) p-output curves and (b) p-transfer curves of the melt crystallized Brush 4 and (c) measured OFET hole mobilities $\mu$ plotted against the molecular weight $M_{n, M A L D I}$ of the linear P3TH respectively the molecular weight $M_{n, M A L D I}$ of the P3HT side-chain in case of the bottlebrush polymers (d) schematic diagram highlighting the dependency of $\mu_{\mathrm{OFET}}$ on the P3HT chain length.

side-chains are necessary if the excellent electronic properties comparable to the linear systems are to be obtained. Moreover, the improved wetting behavior and higher thermal stability of the high molecular weight brushes are of advantage for thin film applications.

\section{Conclusions}

We have demonstrated that there is a strong correlation between the charge carrier mobility of PS-g-P3HT bottlebrushes and their side-chain lengths. In order to investigate the influence of the side-chains on the optical and electronic properties we synthesized a series of PS-g-P3HT bottlebrushes via a grafting-to approach using nitroxide mediated controlled radical polymerization, where only the length of the side-chains was varied. Synthetically we could achieve brushes from 21000 to $144000 \mathrm{~g} \mathrm{~mol}^{-1}$ (SEC), with the highest molecular weight P3HT side-chains being $17400 \mathrm{~g} \mathrm{~mol}^{-1}$ (SEC), which is equivalent to $11400 \mathrm{~g} \mathrm{~mol}^{-1}$ in MALDI-TOF analysis. Charge carrier mobility measurements demonstrated that high molecular weight is necessary to obtain a high charge carrier mobility of $5.0 \times 10^{-2} \mathrm{~cm}^{2} \mathrm{~V}^{-1} \mathrm{~s}^{-1}$ that is competitive with linear P3HTs. We also investigated the structure formation of the grafted polymers in bulk, solution and thin film. The results of DSC, UV-Vis and AFM measurements reveal two important things. The brush polymers with low molecular weight P3HT side-chains do not crystallize and therefore exhibit very poor electronic properties. With an increase of the side-chain length, highly crystalline materials are obtained. These investigations indicate crystallization and aggregation behavior in brushes, which is similar to the linear P3HTs. Lamellar crystallites are for example observed in AFM for the first time for brushes. The excellent thermal stability of the brushes in thin films can be an additional advantage for applications.

\section{Acknowledgements}

The authors acknowledge financial support from DFG (SFB 840), GRK 1640 (DFG) and the Bavarian State Ministry of Education, Science and the Arts (Solar technologies go hybrid). We also thank practical students Alexander Krimalowski, Tobias Jurczyk and Marius Feldmann for their contribution to synthesis.

\section{References}

1 M. Wintermantel, M. Gerle, K. Fischer, M. Schmidt, L. Wataoka, H. Urakawa, K. Kajiwara and Y. Tsukahara, Macromolecules, 1996, 29, 978.

2 M. Zhang and A. H. E. Müller, J. Polym. Sci., Part A: Polym. Chem., 2005, 43, 3461.

3 M. Hu, Y. Xia, G. B. McKenna, J. A. Kornfield and R. H. Grubbs, Macromolecules, 2011, 44, 6935.

4 E. E. Sheina, J. Liu, M. C. Iovu, D. W. Laird and R. D. McCullough, Macromolecules, 2004, 37, 3526. 
5 M. C. Iovu, E. E. Sheina, R. R. Gil and R. D. McCullough, Macromolecules, 2005, 38, 8649.

6 A. Yokoyama, R. Miyakoshi and T. Yokozawa, Macromolecules, 2004, 37, 1169.

7 R. Miyakoshi, A. Yokoyama and T. Yokozawa, J. Am. Chem. Soc., 2005, 127, 17542.

8 R. H. Lohwasser and M. Thelakkat, Macromolecules, 2011, 44, 3388.

9 M. Jeffries-El, G. Sauve and R. D. McCullough, Macromolecules, 2005, 38, 10346.

10 F. P. V. Koch, J. Rivnay, S. Foster, C. Müller, J. M. Downing, E. Buchaca-Domingo, P. Westacott, L. Yu, M. Yuan, M. Baklar, Z. Fei, C. Luscombe, M. A. McLachlan, M. Heeney, G. Rumbles, C. Silva, A. Salleo, J. Nelson, P. Smith and N. Stingelin, Prog. Polym. Sci., 2013, 38, 1978.

11 M. Sommer, S. Huettner and M. Thelakkat, J. Mater. Chem., 2010, 20, 10788.

12 R. H. Lohwasser, G. Gupta, P. Kohn, M. Sommer, A. S. Lang, T. Thurn-Albrecht and M. Thelakkat, Macromolecules, 2013, 46, 4403.

13 S. W. Hong, W. Gu, J. Huh, B. R. Sveinbjornsson, G. Jeong, R. H. Grubbs and T. P. Russell, ACS Nano, 2013, 7, 9684.

14 N. Khanduyeva, V. Senkovskyy, T. Beryozkina, M. Horecha, M. Stamm, C. Uhrich, M. Riede, K. Leo and A. Kiriy, J. Am. Chem. Soc., 2009, 131, 153.

15 N. Khanduyeva, V. Senkovskyy, T. Beryozkina, V. Bocharova, F. Simon, M. Nitschke, M. Stamm, R. Grötzschel and A. Kiriy, Macromolecules, 2008, 41, 7383.

16 P. Paoprasert, J. W. Spalenka, D. L. Peterson, R. E. Ruther, R. J. Hamers, P. G. Evansa and P. Gopalan, J. Mater. Chem., 2010, 20, 2651.

17 D. Meng, J. Sun, S. Jiang, Y. Zeng, Y. Li, S. Yan, J. Geng and Y. Huang, J. Mater. Chem., 2012, 22, 21583.

18 X. Pang, L. Zhao, C. Feng, R. Wu, H. Ma and Z. Lin, Polym. Chem., 2013, 4, 2025.

19 S. Samanta, D. P. Chatterjee, R. K. Layek and A. K. Nandi, J. Mater. Chem., 2012, 22, 10542.

20 S. Ahn, D. L. Pickel, W. M. Kochemba, J. Chen, D. Uhrig, J. P. Hinestrosa, J.-M. Carillo, M. Shao, C. Do, J. M. Messmann, W. M. Brown, B. G. Sumpter and S. M. Kilbey, ACS Macro Lett., 2013, 2, 761.

21 D. v. As, J. Subbiah, D. J. Jones and W. W. H. Wong, Macromol. Chem. Phys., 2016, 217, 403.

22 Z. Bao, A. Dodabalapur and A. J. Lovinger, Appl. Phys. Lett., 1996, 69, 4108.

23 H. Sirringhaus, N. Tessler and R. H. Friend, Science, 1998, 290, 1741.

24 H. Sirringhaus, P. J. Brown, R. H. Friend, M. M. Nielsen, K. Bechgaard, B. M. W. Langeveld-Voss, A. J. H Spiering, R. A. J. Janssen, E. W. Meijer, P. Herwing and P. M. de Leeuw, Nature, 1999, 401, 685.
25 J.-F. Chang, B. Sun, D. W. Breiby, M. M. Nielsen, T. J. Sölling, M. Giles, J. McCulloch and H. Sirringhaus, Chem. Mater., 2004, 16, 4772.

26 R. J. Klein, M. D. McGehee, E. N. Kadnikova, J. Liu, J. M. J. Fréchet and M. F. Toney, J. Am. Chem. Soc., 2006, 128, 3480 .

27 R. J. Kline, M. D. McGehee, E. N. Kadnikova, J. Liu and J. M. J. Fréchet, Adv. Mater., 2003, 15, 1519.

28 A. Zen, J. Pflaum, S. Hirschmann, W. Zhuang, F. Jaiser, U. Asawapirom, J. P. Rabe, U. Scherf and D. Neher, Adv. Funct. Mater., 2004, 14, 757.

29 R. Zhang, B. Li, M. C. Iovu, M. Jefries-EL, G. Sauvé, J. Cooper, S. Jia, S. Tristam-Nagle, D. M. Smilgies, D. N. Lambeth, R. D. McCullough and T. Kowalewski, J. Am. Chem. Soc., 2006, 128, 3480.

30 C. R. Singh, G. Gupta, R. Lohwasser, S. Engmann, J. Balko, M. Thelakkat, T. Thurn-Albrecht and H. Hoppe, J. Polym. Sci., Part B: Polym. Phys., 2013, 51, 943.

31 D. Benoit, V. Chaplinski, R. Braslau and C. J. Hawker, J. Am. Chem. Soc., 1999, 121, 3904.

32 R. H. Lohwasser and M. Thelakkat, Macromolecules, 2012, 45, 3070 .

33 J. Liu, R. S. Loewe and R. D. McCullough, Macromolecules, 1999, 32, 5777.

34 V. V. Rostovtsev, L. G. Green, V. V. Fokin and K. B. Sharpless, Angew. Chem., Int. Ed., 2002, 41, 2596.

35 K. Lorincz, P. Kele and Z. Novak, Synthesis, 2009, 3527.

36 W. E. Garner, K. V. B. Bibber and A. M. King, J. Chem. Soc., 1931, 1533.

37 M. G. Broadhurst, J. Chem. Phys., 1962, 36, 2578.

38 F. P. V. Koch, M. Heeney and P. Smith, J. Am. Chem. Soc., 2013, 135, 13699.

39 C. R. Snyder, R. C. Nieuwendaal, D. M. DeLongchamp, C. K. Luscombe, P. Sista and S. D. Boyd, Macromolecules, 2014, 47, 3942.

40 Z. Wu, A. Petzhold, T. Henze, T. Thurn-Albrecht, R. H. Lohwasser, M. Sommer and M. Thelakkat, Macromolecules, 2010, 43, 4646.

41 J. Balko, R. H. Lohwasser, M. Sommer, M. Thelakkat and T. Thurn-Albrecht, Macromolecules, 2013, 46, 9642.

42 J. Clark, C. Silva, R. H. Friend and F. C. Spano, Phys. Rev. Lett., 2007, 98, 206406.

43 J. Clark, J.-F. Chang, F. C. Spano, R. H. Friend and C. Silva, Appl. Phys. Lett., 2009, 94, 163306.

44 C. Scharsich, R. H. Lohwasser, M. Sommer, U. Asawapirom, U. Scherf, M. Thelakkat, D. Neher and A. Köhler, J. Polym. Sci., Part B: Polym. Phys., 2012, 50, 442.

45 Z. Wu, A. Petzold, T. Henze, T. Thurn-Albrecht, R. H. Lohwasser, M. Sommer and M. Thelakkat, Macromolecules, 2010, 43, 4646. 46 M. Brinkmann and P. Rannou, Macromolecules, 2009, 42, 1125. 